\title{
Validação de comunicação persuasiva audiovisual para redução do consumo de sal por pessoas com insuficiência cardíaca*
}

\author{
Validation of persuasive audiovisual communication to reduce \\ salt consumption by people with heart failure \\ Validación de la comunicación persuasiva audiovisual para reducir \\ el consumo de sal en personas con insuficiencia cardíaca
}

Como citar este artigo:

Sousa MM, Almeida TCF, Gouveia BLA, Freire MEM, Oliveira SHS. Validation of persuasive audiovisual communication to reduce salt consumption by people with heart failure. Rev Esc Enferm USP. 2021;55:e03751. https://doi.org/10.1590/S1980-220X2020006503751

\section{Mailson Marques de Sousa ${ }^{1}$ \\ Taciana da Costa Farias Almeida ${ }^{2}$ \\ Bernadete de Lourdes André \\ Gouveia $^{3}$}

Maria Eliane Moreira Freire ${ }^{4}$

Simone Helena dos Santos Oliveira ${ }^{5}$

* Extraído da tese: "Comunicação persuasiva para motivar a intenção de reduzir o consumo de sal em pessoas com insuficiência cardíaca”, Programa de Pós-Graduação em Enfermagem, Universidade Federal da Paraíba, 2019.

${ }^{1}$ Universidade Federal da Paraíba,

Programa de Pós-Graduação em

Enfermagem, João Pessoa, PB, Brasil.

${ }^{2}$ Universidade Federal de Campina

Grande, Campina Grande, PB, Brasil.

${ }^{3}$ Universidade Federal de Campina Grande, Campus Cuité, Cuité, PB, Brasil.

${ }^{4}$ Universidade Federal da Paraíba, Departamento de Enfermagem Clínica, João Pessoa, PB, Brasil.

${ }^{5}$ Universidade Federal da Paraíba, Escola

Técnica de Saúde, João Pessoa, PB, Brasil.

\section{ABSTRACT}

Objective: To build and validate persuasive audiovisual communication content to reduce salt consumption in the diet of people with heart failure. Method: Methodological study, of a technological method, based on the Theory of Planned Behavior, in which the stages of pre-production, production and post-production were followed. Content validation was carried out with specialists in the field of health and communication. Results: Based on the survey of beliefs related to salt reduction, the video contains 42 screens with three minutes and eleven seconds in duration and has animations and motivational arguments with the aim of positively influencing behavioral intention and modeling negative beliefs in positive ones. In content validation, the judges unanimously agreed with the evaluation criteria for the target audience, language, harmony, figures, narration and persuasion strategy. The Content Validity Index was satisfactory with a 97\% agreement. Conclusion: Persuasive audiovisual communication was considered valid in terms of content, becoming a tool to enhance the reduction of salt consumption in the care of people with heart failure.

\section{DESCRIPTORS}

Persuasive Communication; Heart Failure; Diet, Sodium-Restricted; Video-Audio Media; Social Theory; Behavior. 


\section{INTRODUÇÃO}

A insuficiência cardíaca (IC) é uma doença de natureza sistêmica, considerada como via final da maioria das cardiopatias $^{(1)}$. Entre as medidas de manejo não farmacológico para manutenção da estabilidade clínica da IC, a restrição do consumo de sódio/sal na dieta é uma medida de autocuidado adjuvante para minimização do remodelamento cardíaco dessa população ${ }^{(2)}$. Estudos evidenciam que uma dieta com alto teor de sódio tem sido associada à exacerbação da sintomatologia da IC em decorrência de quadros de congestão pulmonar, favorecendo desfechos clínicos desfavoráveis à vida ${ }^{(3-4)}$.

Diretrizes nacionais e internacionais aconselham a restrição de sódio/sal na dieta da prática clínica, de maneira individualizada, em que devem ser considerados o estágio da doença e a terapêutica farmacológica instituída, a fim de diminuir alterações neuro-hormonais responsáveis por acentuar sintomas de hipervolemia, aumentando o risco de internações hospitalares ${ }^{(5-6)}$. Contudo, essa recomendação apresenta baixa adesão em pacientes com $\mathrm{IC}^{(7)}$.

O estudo BREATHE - Brazilian Registry of Acute Heart Failure, que teve como objetivo traçar o panorama da IC no cenário brasileiro, identificou que $8,9 \%$ das causas de descompensação clínica da doença foram decorrentes do consumo inadequado de sódio e líquidos. $\mathrm{O}$ registro ainda evidenciou que apenas $34,9 \%$ dos pacientes receberam orientações na alta hospitalar sobre a importância de seguir uma dieta com baixo teor de sódio/sal ${ }^{(8)}$.

Nesse sentido, a proposição de intervenções para o fortalecimento/mudança de comportamentos deve ser baseada em teoria para uma melhor compreensão do fenômeno e das variáveis que requerem intervenção. Assim, a Teoria do Comportamento Planejado (TCP) pode auxiliar o enfermeiro para a compreensão dos fatores que determinam o comportamento de redução do consumo de sal, bem como identificar crenças, facilidades e/ou dificuldades para sua execução, oferecendo subsídios para intervir no processo ensino-aprendizagem e motivacional de pessoas com $\mathrm{IC}^{(9)}$.

Acreditando ser a restrição de sódio na dieta um comportamento necessário ao estilo de vida de pessoas com IC, mostra-se relevante a criação de tecnologias de cuidado, que favoreçam a adoção de comportamentos protetores da saúde. Nesse sentido, a comunicação persuasiva apresenta-se como intervenção de potencial êxito nas estratégias voltadas à saúde da população, por ser considerada um meio interventivo utilizado para influenciar normas, atitudes e crenças individuais, que sustentam o comportamento que se pretende modificar ${ }^{(10)}$.

De acordo com a TCP, o foco da comunicação persuasiva é apresentar informações relevantes a um público-alvo, a fim de mudar algumas das suas crenças salientes para formação da intenção comportamental. Assim, intervenções delineadas a partir das crenças comportamentais, normativas ou de controle, podem ter sucesso na produção de mudanças correspondentes em atitudes, normas subjetivas e de controle comportamental percebido. Além disso, essas mudanças podem influenciar ainda mais as intenções na direção desejada do comportamento ${ }^{(9-10)}$.

Pesquisas que empregaram a comunicação persuasiva, com o objetivo de promover a intenção comportamental benéfica à saúde, evidenciaram resultados satisfatórios ${ }^{(11-13)}$. No contexto da IC, ao averiguar o estado da arte, levantaram-se alguns estudos desenvolvidos no cenário internacional fundamentados na TCP, que utilizaram a estratégia de aconselhamento verbal como ferramenta de persuasão para adoção de uma dieta com baixo teor de sódio ${ }^{(14-15)}$.

No cenário nacional, até o desenvolvimento desta pesquisa, não foram identificados estudos sobre a criação de tecnologia do cuidado no formato de comunicações persuasivas dirigidas a pessoas com IC. Portanto, julgou-se pertinente o desenvolvimento dessa tecnologia no formato audiovisual, como proposta inovadora, pela sua versatilidade na combinação de texto, imagem e som, atendendo a necessidade de readaptação das tradicionais estratégias às novas tecnologias de cuidado para essa população.

Com a disponibilização da tecnologia, vislumbra-se a motivação das pessoas com IC a incorporarem a redução de sal na dieta como um comportamento inerente à vida diária, a fim de agregar medidas para alcançar a estabilidade e diminuir as alterações físicas, psicológicas e, consequentemente, os índices de descompensação clínica e morbimortalidade desses indivíduos.

Portanto, o objetivo deste estudo foi construir e validar o conteúdo de uma comunicação persuasiva audiovisual, para reduzir o consumo de sal na dieta de pessoas com IC.

\section{MÉTODO}

\section{TIPO DE ESTUdO}

Estudo metodológico, de natureza tecnológica, realizado em duas etapas: elaboração da comunicação persuasiva de curta duração e validação de conteúdo com juízes especialistas, realizado no período de maio a setembro de 2018.

\section{$1^{a}$ ETAPA: ELABORAÇÃO DA COMUNICAÇÃO PERSUASIVA NO FORMATO AUDIOVISUAL, DE ACORDO COM AS RECOMENDAÇÕES PROPOSTAS POR FLEMING: PRÉ-PRODUÇÃO, PRODUÇÃO E PÓS-PRODUÇÃO (16)}

De modo geral, a TCP indica que a comunicação persuasiva deve ser direcionada a crenças específicas que afetam a intenção comportamental, visando motivar e reforçar positivamente a execução do comportamento. Assim, na fase de pré-produção foi elaborada a comunicação persuasiva textual, a partir das crenças comportamentais, normativas e de controle relacionadas ao comportamento, que foram analisadas e publicadas em estudo prévio $^{(17)}$.

É recomendado utilizar informações precisas e relevantes que possam agregar e/ou influenciar mudanças de crenças que promovam o comportamento desejado ${ }^{(10)}$. Assim, optou-se por utilizar o termo "sal", por ser facilmente apreendido pelo público-alvo e ser a principal fonte na qual se encontra o sódio utilizado para o preparo da dieta. Em adição, realizou-se consultas em diretrizes ${ }^{(5)}$, $^{\text {guidelines }}{ }^{(6)}$ e na 
literatura científica ${ }^{(18)}$ sobre as orientações educativas para redução do consumo de sódio. A partir da consideração destes aspectos, das crenças eliciadas ${ }^{(17)}$ e das orientações propostas para elaboração de comunicações persuasivas, em consonância ao referencial teórico adotado ${ }^{(9-10)}$, o roteiro foi produzido (Script).

Reuniões científicas foram realizadas no Grupo de Pesquisa em Doenças Crônicas com a participação de três enfermeiros com experiência clínica em enfermagem cardiovascular e, ainda, foram ouvidos três psicólogos clínicos com experiência na TCP e em estudos atinentes à comunicação persuasiva, para analisar a capacidade de persuasão do material escrito e permitir o seu refinamento. $\mathrm{Na}$ sequência das reuniões com os membros do grupo, aspectos da narração e elementos audiovisuais, que seriam empregados na comunicação persuasiva, deram conclusão ao Storyboard.

A etapa de produção do vídeo destina-se a gravação das cenas descritas pelo Storyboard ${ }^{(16)}$ e contou com a colaboração de um profissional da área de mídias digitais para confecção das ilustrações, design e layout. A produção das telas foi realizada por meio do programa Adobe Photoshop ${ }^{\oplus}$. Já a narração foi realizada por uma jornalista com experiência em locução, utilizando linguagem congruente ao roteiro previamente elaborado.

A pós-produção do vídeo foi efetuada pelo pesquisador responsável e o profissional de mídias digitais, com auxílio do programa Adobe Premiere ${ }^{\varpi}$ Nessa etapa, foi realizada a edição das telas e animações por meio do Adobe After Effects ${ }^{\circledR}$. A trilha sonora, de domínio público, foi disponibilizada pelo canal Youtube audio library, empregando-se os efeitos sonoros: Natural e White Hats. Nessa fase, reunião com os membros do grupo de pesquisa permitiram orientar ajustes finais antes da etapa de validação.

\section{2a ETAPA: VAlidAÇÃO DE CONTEÚDO}

Após a estruturação, a comunicação persuasiva audiovisual foi submetida à validação de conteúdo por uma equipe multiprofissional em saúde e por profissionais técnicos da área de comunicação. Optou-se pela escolha de uma equipe multiprofissional em consonância com as recomendações da Sociedade Brasileira de Cardiologia, para que o acompanhamento da IC empregue uma abordagem multidisciplinar contínua ${ }^{(5)}$. Os juízes especialistas foram recrutados por meio de amostragem não probabilística intencional, do tipo bola de neve. Realizaram-se buscas na Plataforma Lattes para seleção dos especialistas da área da saúde e da comunicação.

Os critérios de seleção dos profissionais de saúde foram: ser no mínimo especialista na área de cardiologia, ter dois anos de experiência na assistência de pacientes com IC e publicação na área relacionada à $\mathrm{IC}$ ou com conhecimento no referencial teórico metodológico (TCP). Para os juízes técnicos foram considerados: ter no mínimo especialização na área de comunicação e experiência de um ano na elaboração de recursos audiovisuais (vídeos, aplicativos e mídias digitais).

Identificou-se 17 potenciais especialistas. Em seguida, foram encaminhadas cartas-convite de participação no estudo. Após o convite, onze profissionais aceitaram participar da etapa de validação, sendo oito da área da saúde e três da comunicação. Na sequência, foram enviados por e-mail os seguintes documentos: os Termos de Consentimento Livre Esclarecido (TCLE) em duas vias, a versão do vídeo e o instrumento de coleta de dados. Este continha informações sobre o objetivo do estudo, breve descrição do referencial teórico metodológico que fundamentou a construção do material e ficha de avaliação da comunicação persuasiva audiovisual. Solicitou-se o retorno do material no prazo de 20 dias, sendo prorrogado por mais 15 a 30 dias, de acordo com a solicitação do juiz.

A ficha de avaliação, construída pelo pesquisador, continha itens avaliativos do recurso audiovisual que abrangeram: conteúdo e linguagem para o público-alvo da comunicação, ilustrações, figuras, narração, tempo de duração e potencial persuasivo. Os itens foram respondidos por meio de escala do tipo Likert de quatro pontos, sendo 1 = discordo totalmente; 2 = discordo; 3 = concordo; 4 = concordo totalmente. Ao final da avaliação um espaço foi destinado para sugestões, críticas e opinião geral do vídeo.

\section{ANÁLISE E TRATAMENTO DOS DADOS}

Para analisar os resultados da validação pelos juízes especialistas, utilizou-se o Índice de Validade de Conteúdo (IVC), através da concordância dos itens individualmente (Item-Level Content Validity Index I-CVI), expressa pela fórmula: número de especialistas que avaliaram os itens com notas 3 ou 4, dividido pelo número total de juízes. Para classificar o item como válido é desejável um valor igual ou superior a $0,90^{(19)}$. Os critérios avaliados que obtiveram IVC $<0,90$ foram reformulados com base na sugestão dos especialistas e literatura científica. Para avaliar o recurso audiovisual como um todo se utilizou o critério de concordância, que foi obtido ao dividir o número total de itens considerados como válidos pelos juízes, pelo número total de itens. Considerou-se o vídeo como validado quando obtida concordância de no mínimo $90 \%{ }^{(19)}$.

As informações obtidas sobre os profissionais participantes e as respostas foram organizadas no software Excel para Windows, seguidas de análise descritiva, com cálculo de frequências absolutas e relativas, além das medidas de tendência central (média) e de dispersão (desvio padrão).

\section{Aspectos éticos}

O presente estudo integra a pesquisa intitulada: "Efeito de intervenção persuasiva na intenção comportamental de redução do consumo de sal em pacientes com insuficiência cardíaca", aprovada no Comitê de Ética em Pesquisa, sob o Parecer no 2.406.616/2017.

\section{RESULTADOS}

Os argumentos elaborados para modelar os construtos da TCP e suas respectivas crenças estão apresentados no Quadro 1. 
Quadro 1 - Argumentos elaborados com base nas crenças de pessoas com IC relacionadas à redução do consumo de sal na dieta João Pessoa, PB, Brasil, 2018.

\begin{tabular}{|c|c|c|}
\hline Construto & Crenças salientes & Argumentos motivacionais \\
\hline \multirow[b]{2}{*}{ Atitude } & Crenças comportamentais & \multirow{2}{*}{$\begin{array}{l}\text { - Importância da redução do consumo de sal na dieta, para manutenção da saúde } \\
\text { e qualidade de vida } \\
\text { - Associação do consumo excessivo de sal com a piora da sintomatologia da } \\
\text { insuficiência cardíaca } \\
\text { - Ajuste aos novos hábitos imperativos da dietoterapia } \\
\text { - Adaptação à palatabilidade dos alimentos com baixo teor de sal }\end{array}$} \\
\hline & $\begin{array}{l}\text {-Manter a saúde } \\
\text {-Evitar a piora dos sintomas } \\
\text {-Diminuir o apetite } \\
\text {-Mudança no gosto dos alimentos } \\
\text { - Hábito em usar o sal }\end{array}$ & \\
\hline \multirow[b]{2}{*}{ Norma subjetiva } & Crenças normativas & \multirow[b]{2}{*}{$\begin{array}{l}\text { - Importância da família na escolha e preparo dos alimentos com pouca } \\
\text { quantidade de sal } \\
\text { - A construção de rede de apoio para fortalecer e motivar a adesão à dieta prescrita }\end{array}$} \\
\hline & $\begin{array}{l}\text {-Esposa(o) } \\
\text {-Filhos(as) } \\
\text {-Irmãos(ãs) }\end{array}$ & \\
\hline \multirow[b]{2}{*}{$\begin{array}{l}\text { Controle } \\
\text { comportamental } \\
\text { percebido }\end{array}$} & Crenças de controle & \multirow[b]{2}{*}{$\begin{array}{l}\text { - Estratégias para facilitar a substituição do sal } \\
\text { - Táticas para acentuar o sabor da dieta e melhorar a palatabilidade } \\
\text { - Orientar o responsável pelo preparo das refeições a reduzir quantidade de sal } \\
\text { - Reforço para capacidade de seguir uma dieta com pouco sal }\end{array}$} \\
\hline & $\begin{array}{l}\text {-Preparo das refeições pela esposa } \\
\text {-Preparo das refeições separadamente } \\
\text {-Família ajuda a lembrar } \\
\text {-Perda do sabor da dieta } \\
\text {-Falta de controle no preparo das refeições }\end{array}$ & \\
\hline
\end{tabular}

Fonte: Elaborado pelos autores.

O roteiro incluiu uma apresentação institucional, definição da IC, informações sobre dados epidemiológicos, principais sinais e sintomas da IC, comunicações persuasivas ressaltando as vantagens sobre a redução do consumo de sal e as consequências da sua ingestão excessiva, a importância dos referentes sociais como agentes de apoio para seguir uma dieta com baixo consumo de sal, as medidas que podem facilitar a redução do sal, orientações para substituição do sal e, por fim, mensagens de motivação para a adoção e capacidade de seguir uma dieta com baixo teor de sal.

Participaram da etapa de validação de conteúdo oito juízes especialistas, com experiência assistencial de no mínimo dois anos na área do estudo, sendo seis do sexo feminino e dois do sexo masculino, com média de idade 42,8 anos, desvio padrão de 11,8 e variação de 29 a 60 anos. Cincos juízes eram doutores na área da saúde, dois especialistas em cardiologia e um mestre em educação. Quanto à formação profissional, cinco eram enfermeiros, dois médicos cardiologistas e um nutricionista.

No tocante aos especialistas técnicos, profissionais da comunicação audiovisual, os três selecionados eram do sexo masculino, com média de idade de 33,6 anos, desvio padrão de 6,6 anos e variação de 26 a 38 anos de idade. Dois participantes eram mestres, que atuavam como professores de ensino superior na área de Mídias Digitais, e um especialista em comunicação e marketing, que atuava em agência de publicidade.

Quanto à avalição dos juízes, entre os critérios avaliativos, nenhum assinalou a resposta "discordo totalmente". Nas questões relacionadas ao conteúdo para o público-alvo, linguagem, harmonia, ilustrações, narração, estratégia de persuasão e tecnologia de cuidado para os profissionais de saúde, observa-se unanimidade de concordância entre os especialistas, conforme evidenciado na Tabela 1.

Tabela 1 - Concordância entre os especialistas acerca do conteúdo da comunicação persuasiva - João Pessoa, PB, Brasil, 2018.

\begin{tabular}{lcc}
\hline Critérios avaliados & I-CVI $^{*}$ & 1,00 \\
\hline 1. O conteúdo é apropriado para o público-alvo & 1,00 & 0,90 \\
2. A linguagem é de fácil compreensão & 1,00 \\
3. As cores e formas das ilustrações estão adequadas & 1,00 \\
4. A disposição das figuras está em harmonia com o texto & 1,00 \\
5. As ilustrações são relevantes para a compreensão do conteúdo & 0,90 \\
6. A narração está adequada para o conteúdo & 0,90 \\
7. A comunicação é expressa de modo persuasivo & 1,00 \\
8. O tempo de duração é satisfatório & 1,00 \\
9. A comunicação contribui como estratégia de persuasão para mudança de crenças e comportamentos protetores & \\
10. Pode ser utilizado como tecnologia de cuidado pelos profissionais de saúde &
\end{tabular}

\section{${ }^{*}$ Item-Level Content Validity Index}

Pode-se observar que não houve unanimidade nas categorias em relação às cores e às formas das ilustrações, ao tempo de duração e à persuasão. Embora o índice de concordância para os itens tenha sido satisfatório, os pesquisadores consideraram a sugestão pertinente e pequenos ajustes foram realizados nas telas para aprimoramento do material. Optou-se por atender o argumento de um juiz sobre a mudança da cor de fundo vermelho em duas telas e uma adaptação na ilustração que simulava o preparo da refeição pela esposa. Dessa forma, o vídeo foi desenvolvido com 42 telas. Na Figura 1 são ilustradas algumas das telas produzidas. 


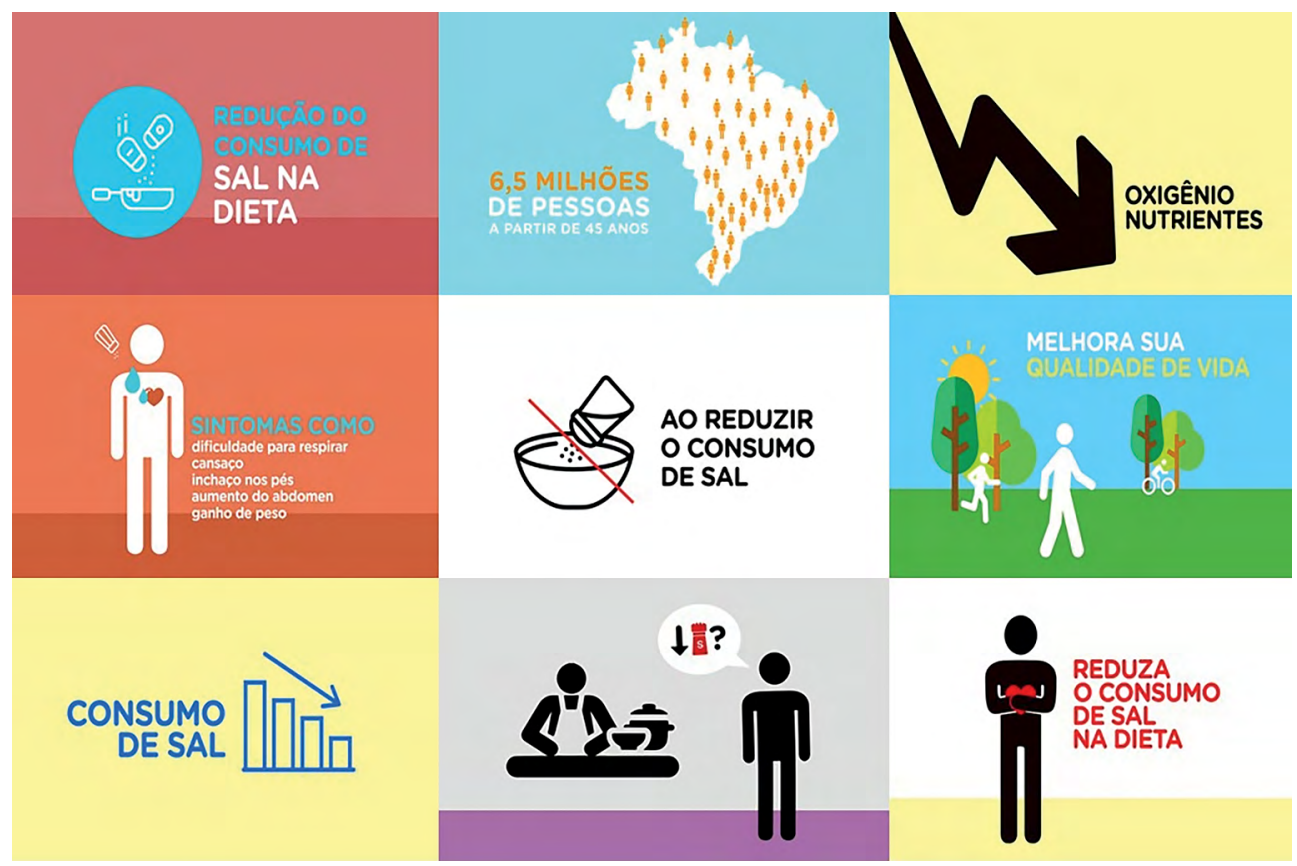

Figura 1 - Resumo das telas produzidas para o recurso audiovisual - João Pessoa, PB, Brasil, 2018.

Apenas um juiz assinalou que a comunicação não estava expressa de maneira persuasiva, atribuindo na avaliação pela escala Likert a pontuação "discordo". Contudo, não indicou quais pontos deveriam ser ajustados e, por isso, não foi possível ponderar sua avaliação na perspectiva de ajustes. Acredita-se que este resultado pode ter ocorrido por incompreensões sobre a estratégia de persuasão e/ou referencial teórico.

Em relação à duração do vídeo, é oportuno destacar que na etapa de pós-produção constatou-se que a narração necessitava de períodos de pausas mais definidos, para melhor compreensão e fluidez da comunicação persuasiva. Sendo assim, a versão inicial do vídeo, que totalizava dois minutos e cinquenta segundos, foi ampliada para três minutos e onze segundos, na perspectiva de melhor se adequar às características da população-alvo.

Os resultados relativos à duração evidenciam IVC suficiente $(\geq 0,90)$, entretanto, um juiz da área da saúde sugeriu a redução do tempo do vídeo, sugestão não acatada em virtude de não fazer parte das recomendações dos demais juízes e, ainda, pela possibilidade de fragilizar a comunicação com a supressão de informações relevantes ao contexto. Ademais, ressalta-se as características inerentes à população-alvo do estudo (baixa escolaridade e idosos), que requerem cuidado na emissão de comunicações em ritmo e clareza conducentes às condições cognitivas, visuais e auditivas decorrentes do processo de envelhecimento.

Outro juiz, técnico em mídias digitais, argumentou que para reprodução em outras plataformas (televisão) o vídeo deveria ser reduzido. Entretanto, a sugestão não foi acatada em virtude de que o objetivo é que o conteúdo seja disseminado em ambiente online e em serviços de saúde, como uma das estratégias para favorecer a adesão terapêutica e, nesse sentido, priorizou-se garantir sua clareza em um ritmo mais adequado ao público-alvo.
A substituição das animações construídas para apresentar os sinais e sintomas da IC por imagens de pessoas reais com quadros de descompensação clínica foi sugerida por um juiz técnico em mídias digitais, acreditando possuírem maior capacidade de persuasão.

Em linhas gerais, o recurso audiovisual evidenciou unanimidade entre os especialistas de conteúdo e técnicos em relação à clareza e à pertinência do material, sendo considerado adequado para ser utilizado como tecnologia de cuidado adjuvante na prática clínica assistencial de profissionais de saúde, em especial do enfermeiro, no manejo da IC.Em face aos bons resultados obtidos da validação de conteúdo, houve apenas um ciclo de avaliação dos especialistas, com concordância de $97 \%$. A versão final da comunicação persuasiva pode ser acessada no Youtube ${ }^{\varpi}$, no canal do Laboratório de Tecnologias do Cuidar vinculado a Universidade Federal da Paraíba, disponível no seguinte endereço: https://youtu. be/GNqfSrgdX4I.

\section{DISCUSSÃO}

O desenvolvimento de Tecnologias de Informação e Comunicação (TICs) na área de enfermagem vem crescendo ao longo das últimas décadas, contribuindo para o aprimoramento de conhecimentos e habilidades no processo de ensino-aprendizagem, além de se tornarem ferramentas para auxiliar o processo de trabalho do enfermeiro, sem fronteiras geográficas ${ }^{(20)}$.

Os recursos audiovisuais constituem-se em ferramentas tecnológicas utilizadas para fomentar informações e reflexões, por meio de mensagens dinâmicas e interativas, incentivando a mudança de crenças, hábitos e comportamentos prejudiciais à saúde ${ }^{(21)}$. Um estudo de revisão identificou que dentre as funcionalidades dos recursos audiovisuais, a função motivadora permite a reflexão de um grupo sobre um tema, 
despertando maior interesse para discussões. Os autores destacam que o uso de imagens e som deve ser explorado como meio para exemplificar situações atitudinais, com o propósito de adotar novos e satisfatórios padrões comportamentais ${ }^{(22)}$.

Considerando o exposto, o recurso audiovisual desenvolvido neste estudo foi avaliado por profissionais da área da saúde, incluindo especialistas na área de cardiologia, assim como por profissionais com formação/atuação em mídias digitais. Nesta pesquisa, por meio de critérios de clareza para público-alvo, linguagem, harmonia, ilustrações e relevância do conteúdo, os especialistas consideraram o vídeo adequado para aplicação como tecnologia voltada ao cuidado de pacientes com IC.

$\mathrm{Na}$ construção de recursos audiovisuais recomenda-se não utilizar cores vibrantes ${ }^{(23)}$. Assim, para melhorar os aspectos estéticos e dar maior visibilidade textual, foram substituídas as cores vermelha, no fundo de uma tela, e amarela, de um texto, sendo utilizados cores frias e tons pastéis que transmitissem leveza, bem-estar e sutileza às imagens.

No que tange a crença de controle preparo das refeições pela esposa, foi sugerido que não estivesse vinculado somente à esposa, mas a qualquer pessoa responsável pelo preparo no ambiente familiar (esposo(a), filhos(as), cuidadores e secretários do lar). Diante disso, os pesquisadores optaram por utilizar um ícone neutro que não caracterizava um gênero específico no preparo das refeições.

Pesquisa realizada nos Estados Unidos da América, ancorada na TCP, evidenciou a norma subjetiva (pressão social percebida) como preditora de uma dieta com baixo teor de sódio em pessoas com IC ( $p=0,032)$. Os autores recomendam que os profissionais de saúde expressem claramente sua opinião sobre o seguimento da dieta, apoiando e motivando pessoas com IC a reduzirem o consumo de sódio dietético, a fim de evitar quadros de exacerbação da doençç ${ }^{(24)}$. Nesse contexto, reforça-se a necessidade de incluir referentes sociais nas estratégias educativas acerca da escolha/substituição de alimentos adequados para o preparo da dieta com baixo teor de sal, de forma que eles possam configurar-se como elos positivos na motivação para a adesão ao comportamento.

No áudio empregado, decidiu-se não utilizar uma linguagem com termos técnicos, bem como expressões populares. Optou-se por uma locução com uma linguagem de fácil entendimento, compatível ao nível de escolaridade do público-alvo, pausada, com entoação suave e em conformidade à comunicação textual elaborada durante a pré-produção. Outro recurso utilizado foi a trilha sonora subdividida para os momentos de informação sobre a IC e motivacional para o reforço positivo. O fundo musical apresentava melodia agradável, de alerta e de aprovação.

Os juízes da área de comunicação, com experiência em mídias digitais, apresentaram manifestações alinhadas aos profissionais de saúde, ao consideraram o vídeo com linguagem adequada para o público-alvo, compreensivo, com layout, animações, ilustrações, dinamismo e tempo de duração satisfatórios.

Sobre esse aspecto, a literatura sugere que o vídeo possua uma linguagem simples, contribuindo para captar a atenção do telespectador, deixando o conteúdo claro e objetivo, capaz de atingir o público-alvo e sensibilizar grupos sociais para influenciar atitudes e comportamentos desejáveis ${ }^{(25-26)}$.
No tocante à capacidade de persuasão, apenas um juiz argumentou que o vídeo não apresentava esta característica, com a justificativa de utilizar imagens reais de pessoas em situações clínicas de descompensação para este fim. No entanto, essa sugestão não foi acatada, ponderando-se que o vídeo tem como proposta motivar de forma lúdica a redução do consumo de sal na dieta, através de reforços positivos, e não utilizar o recurso midiático elaborado como mecanismo para despertar ou fortalecer a sensação de medo ou insegurança.

Dessa forma, entende-se que uma comunicação positiva pode fortalecer e apoiar a intenção favorável para realização do comportamento, evitando situações de aversão ao conteúdo, cenas e argumentos utilizados em mensagens de cunho negativo. Assim, destaca-se a relevância da comunicação persuasiva como ferramenta para influenciar intenções comportamentais para a tomada de decisão, modelando crenças negativas, as quais ofereçam risco à saúde, em crenças positivas.

No critério tempo de duração do vídeo, um juiz argumentou que poderia ser reduzido para apresentação. Após reflexões, optou-se por não reduzir, considerando que o tempo de três minutos e onze segundos de duração mostra-se aceitável para exposição do conteúdo e dentro do recomendado no contexto da saúde. Aconselha-se que vídeos direcionados à promoção da saúde com foco na mudança de comportamentos não ultrapassem o tempo de 20 minutos, para não gerar dispersão e desinteresse pelas informações ${ }^{(21)}$.

Assim, acredita-se que o tempo de duração é satisfatório e a sua redução poderia fragilizar a comunicação elaborada, retirando aspectos relevantes à influência comportamental e ao entendimento da temática. Ademais, o vídeo foi gerado com a finalidade de aplicação em serviços de saúde, com a proposta de esclarecer dúvidas e motivar o público-alvo para o autocuidado. Em acréscimo, estará disponível online ou poderá ser enviado via aplicativo para pessoas com IC e, portanto, o tempo de reprodução é compatível aos contextos de aplicação aos quais se destina.

É relevante destacar que estudos que utilizaram recursos audiovisuais como tecnologia de cuidado, em diferentes contextos das afecções cardiovasculares, evidenciaram resultados positivos. Como exemplo, uma pesquisa que verificou a efetividade do uso de recursos audiovisuais para orientação pré-operatória de revascularização do miocárdio, sobre o conhecimento relacionado ao procedimento cirúrgico, quando comparado à orientação usual. Constatou que o grupo de intervenção apresentou $7,20 \pm 1,56$ acertos, enquanto que o grupo controle teve média de 2,71 $\pm 1,96$ acertos, com diferença estatisticamente significativa $(\mathrm{p}<0,001)$. Os resultados levaram os pesquisadores a concluírem que orientações realizadas com recursos audiovisuais são mais efetivas para fixação do conhecimento, quando comparadas às orientações verbais ${ }^{(27)}$.

Estudos de desenvolvimento de tecnologia audiovisual têm agregado a validação de conteúdo com especialistas, no sentido de produzir uma ferramenta útil, de fácil aplicabilidade, adequada e compreensiva para o público-alvo ao qual se destinam ${ }^{(21,25,28)}$. Assim como nesta pesquisa, essa etapa permitiu agregar uma ampla variedade de conhecimentos e maior qualidade no refinamento do material produzido, colaborando para alcançar o objetivo proposto. 
Dessa forma, acredita-se que para a prática de enfermagem, esse produto tecnológico servirá como recurso adicional no sentido de motivar a adoção de comportamentos protetores da saúde de forma dinâmica e lúdica, contribuindo para a formação de novas crenças e modulação de crenças negativas em positivas, de maneira reflexiva.

Ressalta-se que o desenvolvimento e a aplicabilidade desse recurso audiovisual alinham-se às ações propostas no Plano de Enfrentamento para Controle de Doenças Crônicas, em especial as cardiovasculares, no eixo de promoção da saúde, que consiste em promover estratégias educativas para reduzir o sal adicionado no preparo das refeições e escolha de produtos para o consumo com menor teor de sódio ${ }^{(29)}$.

Como possível limitação deste estudo, aponta-se a ausência de validação de conteúdo do recurso audiovisual com a população-alvo. Entretanto, o conteúdo da comunicação emergiu das crenças emitidas por representantes desta população e houve a preocupação de agregar ao recurso linguagem compatível aos resultados obtidos no levantamento das crenças $^{(17)}$. A partir dessa compreensão, o desenvolvimento do produto tecnológico teve seu desenvolvimento apoiado em conhecimento teórico e empírico, incorporando referencial teórico conducente ao objeto de investigação, à contribuição de especialistas de diferentes áreas de formação/atuação e às crenças comportamentais, normativas e de controle sobre o comportamento da população-alvo.

Ressalta-se que a etapa seguinte desta pesquisa almeja avaliar a aplicabilidade e aceitabilidade da comunicação persuasiva, como estratégia de intervenção na prática clínica, através de estudo piloto, quase-experimental, com o objetivo de modular os determinantes da intenção comportamental relacionada à redução do consumo de sal em pessoas com IC

Sugere-se que pesquisas futuras possam ser desenvolvidas em outros cenários do país, em virtude da diversidade cultural alimentar, a fim de levantar novas crenças ou ampliar os aspectos contemplados neste estudo. Ponderando que os argumentos selecionados para a produção do vídeo foram construídos com base nas crenças da realidade regional, o que pode não expressar as crenças e hábitos alimentares de outras regiões.

Ademais, recomenda-se a construção de novas mídias que contemplem os demais aspectos atrelados ao manejo farmacológico e não farmacológico da IC, para promover o autocuidado, a fim de alcançar e manter a estabilidade clínica da doença.

\section{CONCLUSÃO}

A comunicação persuasiva de curta duração do tipo audiovisual, direcionada à redução do consumo de sal na dieta de pessoas com IC, foi considerada válida quanto ao conteúdo pelos especialistas da área de cardiologia e de comunicação. $\mathrm{O}$ vídeo possui duração de três minutos e onze segundos e é constituído de 42 telas, além de estar disponível on-line e gratuitamente. Assim, espera-se que de forma atrativa e dinâmica possa ser utilizado como ferramenta adjuvante motivacional na prática clínica do enfermeiro no atendimento de pessoas com IC.

\section{RESUMO}

Objetivo: Construir e validar o conteúdo de comunicação persuasiva audiovisual, para reduzir o consumo de sal na dieta de pessoas com insuficiência cardíaca. Método: Estudo metodológico, de natureza tecnológica, ancorado na Teoria do Comportamento Planejado, no qual foram seguidas as etapas de pré-produção, produção e pós-produção. Realizou-se validação de conteúdo com especialistas na área da saúde e comunicação. Resultados: Com base no levantamento de crenças relacionadas à redução de sal, o vídeo dispõe de 42 telas, três minutos e onze segundos de duração e contém animações e argumentos motivacionais com o objetivo de influenciar positivamente a intenção comportamental e modelar crenças negativas em positivas. Na validação de conteúdo, os juízes concordaram, em unanimidade, com os critérios de avaliação para o público-alvo, linguagem, harmonia, ilustrações, narração e estratégia de persuasão. O Índice de Validade de Conteúdo mostrou-se satisfatório com concordância de $97 \%$. Conclusão: A comunicação persuasiva, no formato audiovisual, foi considerada válida quanto ao conteúdo, tornando-se uma ferramenta para potencializar a redução do consumo de sal no cuidado de pessoas com insuficiência cardíaca.

\section{DESCRITORES}

Comunicação Persuasiva; Insuficiência Cardíaca; Dieta Hipossódica; Mídia Audiovisual; Teoria Social; Comportamento.

\section{RESUMEN}

Objetivo: Construir y validar el contenido de la comunicación audiovisual persuasiva para reducir el consumo de sal en la dieta de personas con insuficiencia cardíaca. Método: Se trata de un estudio metodológico, de carácter tecnológico, basado en la Teoría del Comportamiento Planificado, en el que se siguieron las etapas de preproducción, producción y postproducción. La validación del contenido se realizó con especialistas en el área de la salud y la comunicación. Resultados: Basado en la encuesta sobre las creencias relacionadas con la reducción de la sal, el vídeo tiene 42 pantallas, tres minutos y once segundos de duración, con animaciones y argumentos motivacionales que pretenden influir positivamente en la intención del comportamiento y transformar las creencias negativas en positivas. En la validación del contenido, los jueces estuvieron unánimemente de acuerdo con los criterios de evaluación del público objetivo, el lenguaje, la armonía, las ilustraciones, la narración y la estrategia de persuasión. El índice de Validez del Contenido resultó satisfactorio con un $97 \%$ de concordancia. Conclusión: La comunicación persuasiva, en formato audiovisual, se consideró válida respecto al contenido, convirtiéndose en una herramienta para potenciar la reducción de la ingesta de sal en el cuidado de personas con insuficiencia cardíaca.

\section{DESCRIPTORES}

Comunicación Persuasiva; Insuficiencia Cardíaca; Dieta Hiposódica; Medios Audiovisuales; Teoría Social; Conducta.

\section{REFERÊNCIAS}

1. Groenewegen A, Rutten FH, Mosterd A, Hoes AW. Epidemiology of heart failure. Eur J Heart Fail. 2020;22(8):1342-56. https://doi. org/10.1002/ejhf.1858 
2. Barilli SL, d'Almeida KS, Trojahn MM, Souza GC, Aliti GB, Rabelo-Silva ER. Knowledge, barriers and attitudes toward dietary sodium in patients with decompensated heart failure. J Nurs Educ Pract. 2018;8(1):98-106. https://doi.org/10.5430/jnep.v8n1p98

3. Song EK, Moser DK, Kang SM, Lennie TA. Self-reported adherence to a low-sodium diet and health outcomes in patients with heart failure. J Cardiovasc Nurs. 2016;31(6):529-534. https://doi.org/10.1097/JCN.0000000000000287

4. Colin-Ramirez E, McAlister FA, Woo E, Wong N, Ezekowitz JA. Association between self-reported adherence to a low-sodium diet and dietary habits related to sodium intake in heart failure patients [Internet]. J Cardiovasc Nurs. 2015;30(1):58-65. https://doi.org/10.1097/JCN.0000000000000124

5. Rohde LE, Montera MW, Bocchi EA, Clausell NO, Albuquerque DC, Rassi S, et al. Diretriz brasileira de insuficiência cardíaca crônica e aguda. Arq Bras Cardiol. 2018;111(3):436-539. https://doi.org/10.5935/abc.20180190

6. Ponikowski P, Voors AA, Anker SD, Bueno H, Cleland JG, Coats AJ, et al. 2016 ESC Guidelines for the diagnosis and treatment of acute and chronic heart failure. Eur J Heart Fail. 2016;18(8):891-975. https://doi.org/10.1002/ejhf.592

7. Chan A, Kinsman L, Elmer S, Khanam M. An integrative review: adherence barriers to a low salt diet in culturally diverse heart failure adults [Internet]. Aust J Adv Nurs. 2018 [cited 2020 Jan 10];36(1):37-47. Available from: http://www.ajan.com.au/Vol36/lssue1/4Chan.pdf

8. Albuquerque DC, Souza Neto JD, Bacal F, Rohde LE, Bernardez-Pereira S, Berwanger O, et al. I Brazilian Registry of Heart Failure: clinical aspects, care quality and hospitalization outcomes. Arq Bras Cardiol. 2015;104(6):433-42. https://doi.org/10.5935/abc.20150031

9. Ajzen I. Behavioral interventions based on the theory of planned behavior [Internet]. 2019 [cited 2020 Dec 6]. Available from: https:// people.umass.edu/aizen/pdf/tpb.intervention.pdf

10. Fishbein M, Ajzen I. Predicting and changing behavior: the reasoned action approach. New York: Routledge; 2015.

11. Norman P, Cameron D, Epton T, Webb TL, Harris PR, Millings A, et al. A randomized controlled trial of a brief online intervention to reduce alcohol consumption in new university students: combining self-affirmation, theory of planned behaviour messages, and implementation intentions. Br J Health Psychol. 2018;23(1):108-27. https://doi.org/10.1111/bjhp.12277

12. Shafieinia M, Hidarnia A, Kazemnejad A, Rajabi R. Effects of a theory based intervention on physical activity among female employees: a quasi-experimental study. Asian J Sports Med. 2016;7(2): e31534. https://doi.org/10.5812/asjsm.31534

13. Park J, Kim SH, Kim JG. Effects of message framing and health literacy on intention to perform diabetes self-care: a randomized controlled trial. Diabetes Res Clin Pract. 2020;161:108043. https://doi.org/10.1016/j.diabres.2020.108043

14. Heo S, Moser DK, Lennie TA, Payne-Emerson H, Welch JL, Weaver M. Development and testing of the feasibility and acceptability of a tailored dietary intervention in patients with heart failure. J Cardiovasc Nurs. 2015;30(3):213-21. https://doi.org/10.1097/JCN.0000000000000148

15. Welsh D, Lennie TA, Marcinek R, Biddle MJ, Abshire D, Bentley B, et al. Low-sodium diet self-management intervention in heart failure: pilot study results. Eur J Cardiovasc Nurs. 2013;12(1):87-95. https://doi.org/10.1177/1474515111435604

16. Fleming SE, Reynolds J, Wallace B. Lights... camera... action! a guide for creating a DVD/video. Nurse Educ. 2009;34(3):118-21. https:// doi.org/10.1097/NNE.0b013e3181a0270e

17. Sousa MM, Gouveia BLA, Almeida TFC, Freire MLM, Oliveira SHS. Beliefs of people with salt-related heart failure. Rev Enferm UERJ. 2019;(27): e44197. https://doi.org/10.12957/reuerj.2019.44197

18. Welsh D, Marcinek R, Abshire D, Lennie TA, Biddle M, Bentley B, et al. Theory-based low-sodium diet education for heart failure patients. Home Healthc Nurse. 2010;28(7):432-41. https://doi.org/10.1097/NHH.0b013e3181e324e0

19. Oliveira F, Kuznier TP, Souza CC, Chianca TC. Theoretical and methodological aspects for the cultural adaptation and validation of instruments in nursing. Texto Contexto Enferm. 2018;27(2):e4900016. https://doi.org/10.1590/0104-070720180004900016

20. Pereira FG, Silva DV, Sousa LM, Frota NM. Building a digital application for teaching vital signs. Rev Gaúcha Enferm. 2016;37(2):e59015. https://doi.org/10.1590/1983-1447.2016.02.59015

21. Lima MB, Rebouças CB, Castro RC, Cipriano MA, Cardoso MV, Almeida PC. Construction and validation of educational video for the guidance of parents of children regarding clean intermittent catheterization. Rev Esc Enferm USP. 2017;51:e03273. https://doi.org/10.1590/ s1980-220x2016005603273

22. Sarabia AA, Niño GT, León BDLP, Ortega MI, Knopfler SC, Felipe LM, et al. Audiovisual Aids in nursing education: literature review. Enferm Glob. 2017;16(47):512-38. https://doi.org/10.6018/eglobal.16.3.260621

23. Razera AP, Trettene AS, Mondini CC, Cintra FM, Razera FP, Tabaquim ML. Construction of an educational video on postoperative care for cheiloplasty and palatoplasty. Texto Contexto Enferm. 2019;28:e20180301. https://doi.org/10.1590/1980-265x-tce-2018-0301

24. Wu JR, Lennie TA, Dunbar SB, Pressler SJ, Moser DK. Does the Theory of Planned Behavior predict dietary sodium intake in patients with heart failure? West J Nurs Res. 2017;39(4):568-81. https://doi.org/10.1177/0193945916672661

25. Galindo-Neto NM, Alexandre AC, Barros LM, Sá GG, Carvalho KM, Caetano JA. Creation and validation of an educational video for deaf people about cardiopulmonary resuscitation. Rev Latino-Am Enfermagem. 2019;27:e3130. https://doi.org/10.1590/1518-8345.2765.3130

26. Silva JP, Bernardi FA, Franzon JC, Orlandin L, Ferlin GZ, Pereira Júnior GA. Step-by-step insulin application: making educational videos for patients and caregivers. Esc Anna Nery. 2021;25(1):e20190343. https://doi.org/10.1590/2177-9465-ean-2019-0343

27. Oliveira AP, Souza EN, Pellanda LC. Effectiveness of video resources in nursing orientation before cardiac heart surgery. Rev Assoc Med Bras. 2016;62(8):762-7. https://doi.org/10.1590/1806-9282.62.08.762

28. Interaminense IN, Oliveira SC, Linhares FM, Guedes TG, Ramos VP, Pontes CM. Construction and validation of an educational video for human papillomavirus vaccination. Rev Bras Enferm. 2020;73(4):e20180900. https://doi.org/10.1590/0034-7167-2018-0900

29. Malta DC, Silva Junior JB. [Brazilian Strategic Action Plan to combat chronic non-communicable diseases and the global targets set to confront these diseases by 2025: a review]. Epidemiol Serv Saude. 2013;22(1):151-64. Portuguese. https://doi.org/10.5123/S1679-49742013000100016 